\title{
Desenvolvimento de fonte dingbat: narrativas das mudanças de hábitos causadas pelo isolamento social em tempos de pandemia
}

\author{
Development of dingbat font: narratives of changes in life habits \\ related to social isolation in pandemic times
}

Fernanda Ferreira Santana Silva, João Vitor Barreto de Miranda, Luiza Falcão,
Helena Rugai Bastos

tipografia, dingbats, método projetual, Covid-19, isolamento social

\begin{abstract}
$\mathrm{O}$ artigo relata o processo de criação da fonte dingbat Habitar, inspirada no tema pandemia pelo novo coronavírus (SARS-CoV-2) e desenvolvida na disciplina Desenho de Tipos do Curso de Design da Universidade Federal do Rio Grande do Norte. A fonte sugere o agrupamento dos caracteres para a geração de várias possibilidades de narrativas. O método de Cunha (2019) foi utilizado para o projeto. O dingbat, é composto por 74 símbolos que representam o contexto das mudanças de hábitos causadas pelo isolamento social em tempos de pandemia, e das adaptações abruptas no estilo de vida e na rotina das pessoas. A fonte dingbat desenvolvida possibilita fácil instalação e manuseio em programas de edição de textos. Assim, o projeto pode ser utilizado para compor mensagens e informações em peças de comunicação gráfica em geral.
\end{abstract}

typography, dingbats, design method, Covid-19, social isolation

The article reports the design process of the dingbat font Habitar, inspired by the pandemic theme by the new coronavirus (SARS-CoV-2) and developed in Design of Types discipline of the Design Course of Federal University of Rio Grande do Norte. The font suggests the grouping of characters for the generation of various narrative possibilities. The Cunha (2019) method was used for the project. The dingbat is composed by 74 symbols that represent the context of changes in life habits related to social isolation in pandemic times, and the abrupt lifestyle adjustments in people's routine. The developed dingbat font allows easy installation and handling in text editing programs. Thus, the project can be used to compose messages and information in graphic communication pieces in general.

\section{Introdução}

De acordo com Borges (2011), historicamente, a tipografia tem a função primária de representar graficamente os caracteres presentes na linguagem verbal. Porém, com o passar do tempo e com os avanços tecnológicos, foi possível desenvolver fontes que não atuam apenas como suporte para a língua escrita. Segundo a autora, a tipografia não se restringe aos tipos fundidos, nem à impressão de letras ou ao desenho de caracteres alfabéticos. Os dingbats exemplificam as tipografias que não possuem relação aparente com os caracteres alfabéticos (Borges, 2011). 
O processo projetual descrito neste artigo teve como objetivo o desenvolvimento da fonte dingbat Habitar. O conjunto de caracteres da fonte possibilita a composição de diferentes narrativas e, também, o uso individual dos glifos como elementos gráficos comunicacionais. O projeto foi desenvolvido a partir do método de Cunha (2019), com base em uma pesquisa iconográfica que abordou as mudanças de hábitos causadas pelo distanciamento social, em virtude da pandemia do novo coronavírus (SARS-CoV-2). Nesse contexto, o isolamento apresentou à população uma realidade diferente do habitual, submetendo-a a uma adaptação de hábitos no cotidiano e a busca de outras formas de rotina, comunicação, lazer, higiene, entre outros aspectos.

\section{Fontes dingbats}

Borges (2011) afirma que os dingbats podem ser ornamentos tipográficos, pictogramas ou símbolos, que já existiam e eram utilizados na composição de textos antes mesmo da tipografia se tornar digital. Embora os dingbats não apresentem características alfabéticas visuais, eles possuem uma conexão com o alfabeto latino dada a relação entre a fonte e o teclado, construída através do avanço tecnológico, que possibilitou a organização desses símbolos. Farias (2001) expõe que a tecnologia tornou possível organizar, armazenar e usar esses conjuntos de caracteres que não estão necessariamente relacionados às letras ou aos símbolos tradicionais, da mesma maneira que as fontes letradas.

Mesmo constituindo uma ligação de uso com os caracteres alfabéticos, os dingbats não possuem leitura verbal ou relação fonética, assim, essas fontes configuram símbolos, imagens ou formas visuais que compõem uma linguagem visual. Borges (2011) aponta que os dingbats são signos abertos a uma variedade de significados, o que permite múltiplas interpretações, pois suas características aparentes podem estar associadas a uma gama muito mais ampla de experiências. Desta forma, percebe-se que no projeto de dingbats há espaço para experimentações que explorem novas linguagens, dissociadas da função básica de interface para a linguagem verbal escrita atribuída à tipografia (Borges, 2011).

O projeto da fonte Habitar explorou o dingbat como instrumento para a documentação de hábitos do cotidiano de uma sociedade em tempos de pandemia da COVID-19. Seu processo projetual será relatado a seguir.

\section{Procedimento da disciplina}

O dingbat Habitar foi desenvolvido em 2020 na disciplina Desenho de Tipos, componente curricular optativo do Curso de Design da Universidade Federal do Rio Grande do Norte, ministrado de forma remota pelas professoras Luiza Falcão e Helena Rugai Bastos. A produção do projeto em modalidade remota deu-se pela utilização de ferramentas de videochamada, divisão de tarefas por aptidão e armazenamento compartilhado de arquivos na nuvem. As etapas de base do processo, como a determinação dos parâmetros de construção dos 
desenhos, foram estabelecidas em conjunto pelos integrantes do grupo responsável pelo projeto a fim de garantir coerência formal do conjunto de caracteres.

A partir da temática COVID-19 proposta pelas docentes, a tipografia projetada aborda o contexto das mudanças de hábitos em razão da pandemia do novo coronavírus (SARS-CoV-2) e o consequente isolamento social. A partir da observação das práticas cotidianas no momento de restrição social, houve a determinação de aspectos que foram explorados graficamente a partir da divisão de categorias, a saber: a) hábitos de higiene e uso de equipamento de proteção individual (EPI); b) atividades em geral; e c) atividades em meio digital.

Para o projeto, utilizou-se o método proposto por Cunha (2019). O método, composto por 11 fases, propõe que os desenhos dos caracteres de uma fonte dingbat sejam iniciados a partir da reconstrução inspirada em referências concretas.

\section{Processo metodológico para a criação do dingbat Habitar}

\section{Pesquisa iconográfica}

A pesquisa de imagens buscou referências concretas de artefatos e de situações correspondentes às três categorias mencionadas. A busca foi orientada pela utilização de palavras-chaves a partir do contexto dos hábitos do isolamento social e pela facilidade de reconstrução dos cenários/objetos selecionados em formas sintéticas e em informações visuais concisas.

\section{Categorização e seleção das referências concretas}

As referências foram organizadas nas categorias a) hábitos de higiene e uso de equipamento de proteção individual (EPI); b) atividades em geral; c) atividades em meio digital. Os glifos foram produzidos pela reconstrução de formas inspiradas na seleção das referências concretas, adaptada de processo sugerido por Lima (2009).

\section{Esboços iniciais}

Realizaram-se esboços manuais (Figura 1) com base na pesquisa iconográfica para definição da linguagem visual. Estabeleceu-se o uso de formas simplificadas para garantir a fácil identificação e compreensão dos elementos representados.

Figura 1: Esboços manuais em papel milimetrado. Fonte: autores.

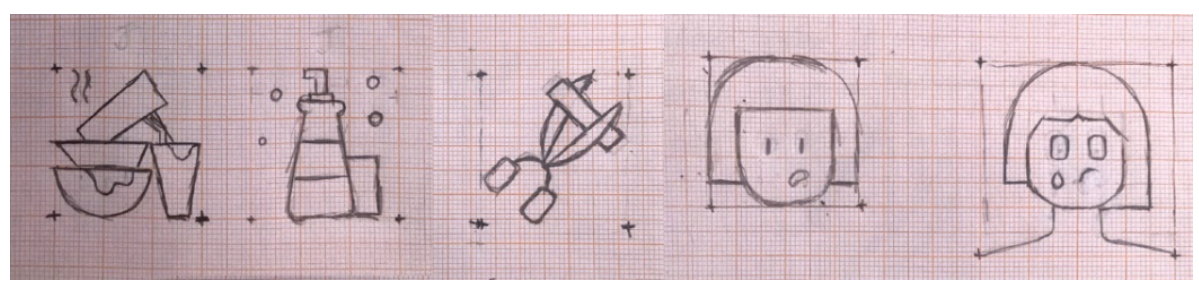


Definição da relação entre a espessura das hastes, proporção vertical e proporção horizontal

Para garantir coerência formal do conjunto de caracteres da fonte, foram definidos os seguintes parâmetros de construção do desenho:

- Proporção entre a espessura das hastes e fundo de 15:1000 pt;

- Peso normal;

- Hastes sem contraste (monolinear);

- Curvas e terminações arredondadas;

- Proporção vertical fixa de 1000 pt;

- Proporções horizontais variadas, de acordo com o conteúdo do caractere.

\section{Regra de funcionamento da caixa-alta e da caixa-baixa}

O projeto prevê o desenvolvimento dos caracteres em quatro níveis, cada um representando um sentido complementar aos outros, possibilitando construção de informações associadas ou narrativas. O funcionamento do conjunto corresponde ao seguinte escopo técnico:

- Caixa baixa e alta: determinou-se uma associação de complemento de sentido estabelecida entre os caracteres de caixa baixa e alta da mesma letra do alfabeto da fonte dingbat projetada, como "a" e "A". As minúsculas, que constituem o primeiro nível, correspondem a uma situação ou ação, e as maiúsculas, que constituem o segundo nível, representam um ou mais objetos associados à situação ou ação do caractere em caixa baixa correspondente;

- Sinais diacríticos: o projeto estabelece o desenho dos caracteres correspondentes apenas às letras do alfabeto acentuadas com acentos agudo, grave, circunflexo, til e cedilha, como usual na língua portuguesa. Essas correspondem à construção do terceiro nível de sentido e representam situações adicionais aos caracteres equivalentes às vogais e ao glifo "c";

- Numerais: na fonte são representados pelo desenho de onomatopeias, funcionando como informações textuais que complementam o sentido de outros caracteres. Também apresentam a versão sobrescrita.

\section{Criação de um grid digital}

Foi criado um grid para construção dos caracteres em software de desenho vetorial com base de 1000x1000 pt, subdividido em módulos que auxiliaram no alinhamento e na proporção de elementos da composição visual de cada caractere.

\section{Desenhos manuais de alguns caracteres}

Foram realizados desenhos manuais dos caracteres base em papel milimetrado a partir de imagens coletadas na pesquisa iconográfica. 


\section{Desenhos digitais de todos os caracteres}

Os desenhos manuais foram transpostos para um software de desenho vetorial de acordo com parâmetros definidos anteriormente (Figura 2). Parte dos glifos dispensaram a etapa de desenhos manuais, pois derivam formalmente de outros caracteres. Assim, foi possível reaproveitar o desenho digital na construção de alguns glifos.

Figura 2: Construção dos caracteres "a" e "j". Fonte: autores.

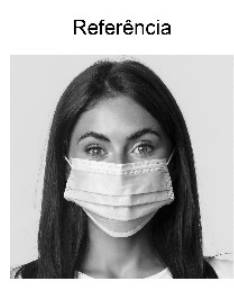

Referência

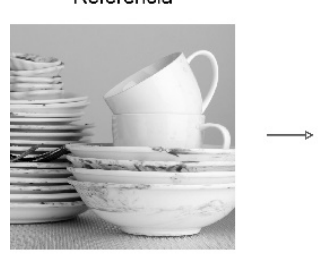

Esboço manual

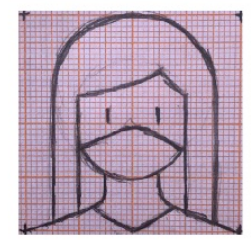

Esboço manual

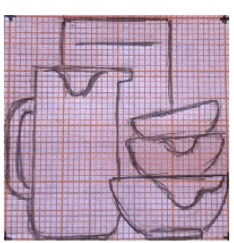

Desenho digital

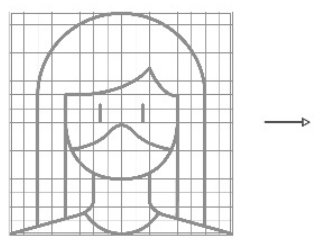

Desenho digital

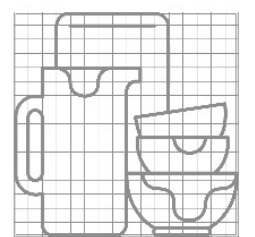

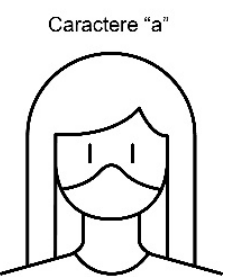

Caractere "j"

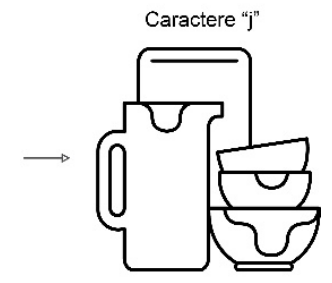

Transposição para o software de geração de fontes

Foi estabelecida a métrica da fonte no software de geração de fontes, além da transposição de todos os desenhos.

\section{Espacejamento lateral}

O espacejamento lateral dos glifos da fonte segue a lógica do método proposto por Tracy (1986) para os caracteres alfabéticos. O autor sugere que o espaço lateral da letra seja estabelecido de acordo com os espaços internos não-preenchidos (Tracy, 1986). Para esta fonte, considerou-se as características particulares dos desenhos de cada glifo para a definição dos seus espaços laterais (Figura 3).

Figura 3: Demonstração de espacejamento lateral. Fonte: autores.

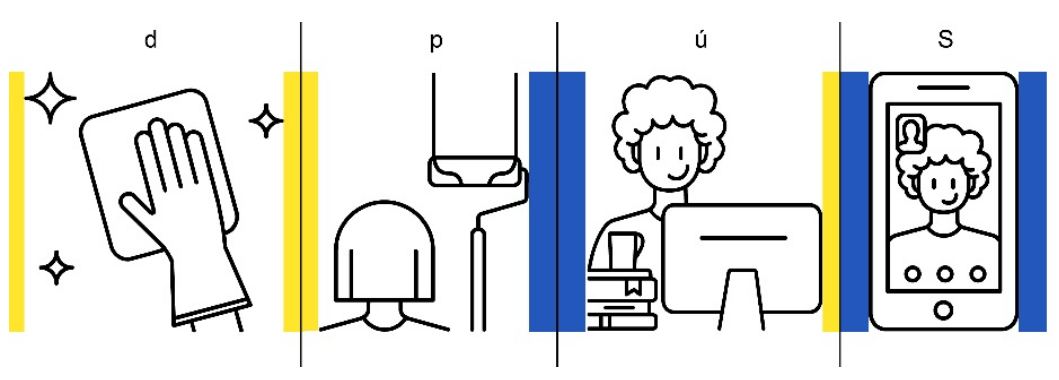




\section{Geração de arquivo fonte}

O arquivo de fonte foi exportado na extensão OpenType (OTF) pelo software de geração de fontes, e instalado para uso. O dingbat Habitar é composto por 74 glifos, que representam as mudanças nos hábitos de higiene e uso de equipamento de proteção individual (EPI), nas atividades em geral, e nas atividades em meio digital, em razão da pandemia do COVID-19 (Figura 4). A fonte foi projetada a partir da relação de complementariedade dos caracteres da caixa alta e baixa, e dos sinais diacríticos (Figura 5).

Vale ressaltar, que o uso dos caracteres em sequência também possibilita a construção de narrativas hipotéticas sobre o contexto de pandemia (Figura 6).

Figura 4: Mapa de caracteres do dingbat Habitar. Fonte: autores.

Caixa baixa

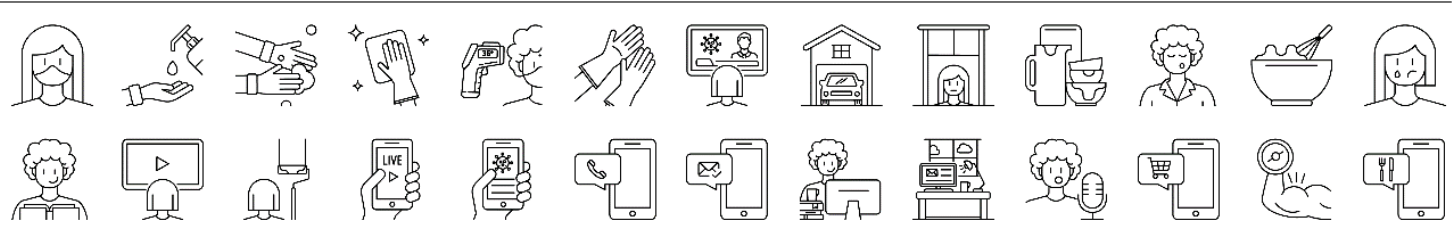

Caixa alta

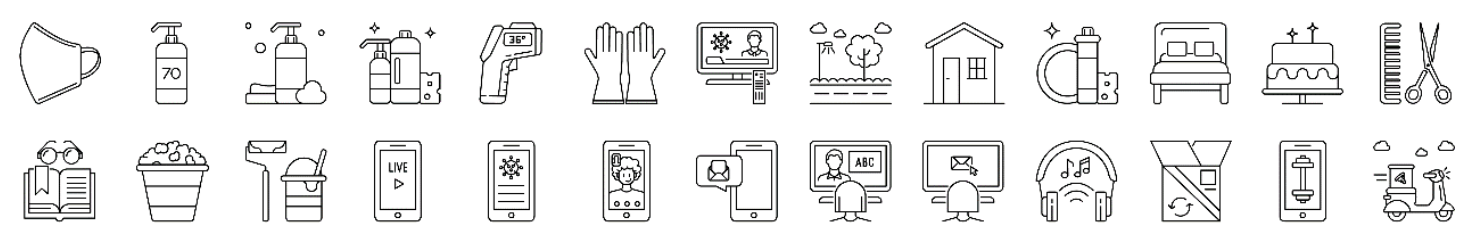

Sinais diacríticos

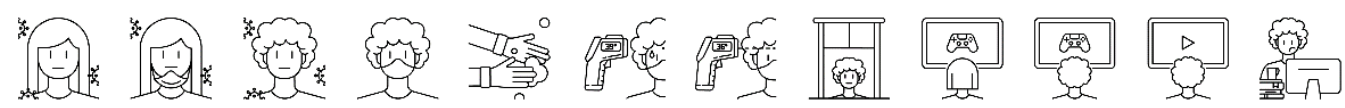

Numerais

AFFFFF ATCHIN BI-BI-BI DIN-DON GRRRRR HAHAHA SHHHHH TIC-TAC URGHHH ZZZZZZ

Figura 5: Relação entre a caixa alta, a caixa baixa e diacríticos. Fonte: autores.
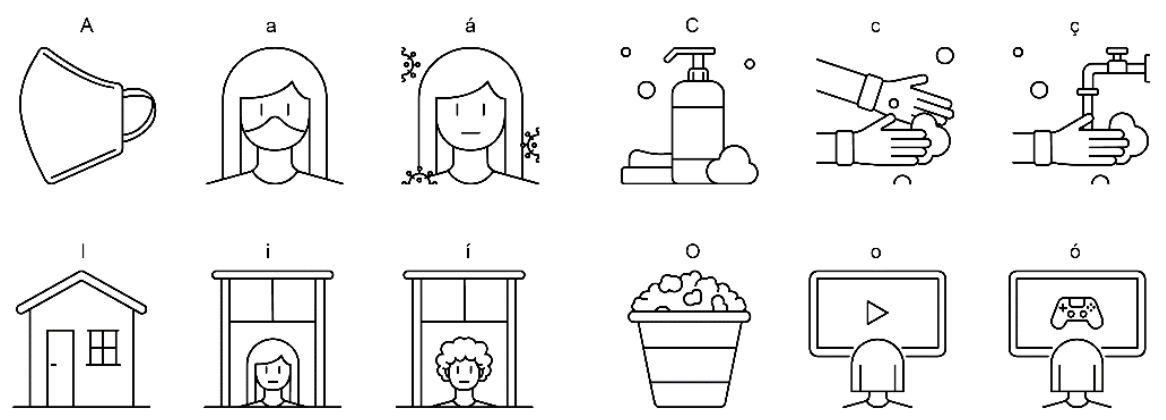

Figura 6: Construção de narrativa hipotética. Fonte: autores.
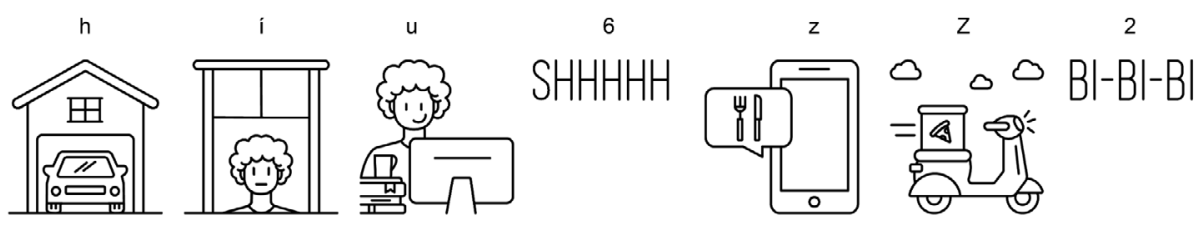


\section{Considerações finais}

O presente artigo relatou o projeto da fonte dingbat Habitar, estruturado em caixa alta, caixa baixa, sinais diacríticos e numerais, a partir da aplicação do método para criação de dingbats proposto por Cunha (2019). A tipografia foi construída em quatro níveis de sentido e de uso, assim os caracteres podem ser utilizados de forma isolada ou conjunta. O agrupamento dos glifos pode criar narrativas sobre novos hábitos em consequência do isolamento social, em razão da pandemia do novo coronavírus (SARS-CoV-2).

A fonte Habitar pode ser facilmente instalada e utilizada em aplicativos de edição de textos, de desenho vetorial e de tratamento de imagens, o que viabiliza sua difusão. Assim, o dingbat pode ser utilizado para compor peças de comunicação gráfica, como elemento decorativo ou de apoio tipográfico, bem como para criação de padrões e texturas. O projeto desenvolvido registra um momento instável e vulnerável da história contemporânea, deve contribuir para a elaboração de novos projetos tipográficos nacionais de dingbats.

\section{Referências}

Borges, P.M. (2011). Fontes Tipográficas Digitais: entre a lógica verbal e a gráfico visual. Revista Galáxia, São Paulo, 22, 262-273.

Cunha, L. F. S. (2019). O desenvolvimento de fontes dingbats como ferramenta para a aprendizagem do processo projetual do design de tipos. Anais do Congresso Internacional de Design da Informação, 9, 917-925. https://doi.org/10.5151/9cidi-congic-2.0193

Farias, P. L. (2001). Fontes que não servem para escrever: algumas considerações sobre o status tipográfico dos Dingbat. Revista da ADG, 23, 49-51.

Lima, F. P. L. de. (2009). O processo de construção das fontes digitais de simulação caligráfica [Dissertação de Mestrado não publicada]. Escola Superior de Desenho Industrial, Universidade Estadual do Rio de Janeiro.

Tracy, W. (1986). Letters of credit: A view of type design. New Hampshire: David R. Godine.

\section{Sobre o(a/s) autor(a/es)}

Fernanda Ferreira Santana Silva, UFRN, Brasil <fernandaffss@outlook.com> João Vitor Barreto de Miranda, UFRN, Brasil <jvbarreto1998@gmail.com> Luiza Falcão, Dra., UFRN, Brasil <luizafsc@gmail.com>

Helena Rugai Bastos, Dra., UFRN, Brasil <helenarugai@gmail.com> 